research

\title{
Adrenocortical LDL receptor function negatively influences glucocorticoid output
}

\author{
Ronald J van der Sluis, Miranda Van Eck and Menno Hoekstra
}

Division of Biopharmaceutics, Cluster BioTherapeutics, Gorlaeus Laboratories, Leiden Academic Centre for Drug Research, Einsteinweg 55, 2333 CC Leiden, The Netherlands

Correspondence should be addressed

to M Hoekstra

Email

hoekstra@lacdr.leidenuniv.nl

\begin{abstract}
Over $50 \%$ of the cholesterol needed by adrenocortical cells for the production of glucocorticoids is derived from lipoproteins. However, the overall contribution of the different lipoproteins and associated uptake pathways to steroidogenesis remains to be determined. Here we aimed to show the importance of LDL receptor (LDLR)-mediated cholesterol acquisition for adrenal steroidogenesis in vivo. Female total body LDLR knockout mice with a human-like lipoprotein profile were bilaterally adrenalectomized and subsequently provided with one adrenal either expressing or genetically lacking the LDLR under their renal capsule to solely modulate adrenocortical LDLR function. Plasma total cholesterol levels and basal plasma corticosterone levels were identical in the two types of adrenal transplanted mice. Strikingly, restoration of adrenal LDLR function significantly reduced the ACTH-mediated stimulation of adrenal steroidogenesis $(P<0.001)$, with plasma corticosterone levels that were respectively $44-59 \%$ lower $(P<0.01)$ as compared to adrenal LDLR negative controls. In addition, LDLR positive adrenal transplanted mice exhibited a significant decrease $(-39 \% ; P<0.001)$ in their plasma corticosterone level under fasting stress conditions. Biochemical analysis did not show changes in the expression of genes involved in cholesterol mobilization. However, LDLR expressing adrenal transplants displayed a marked $62 \%$ reduction $(P<0.05)$ in the transcript level of the key steroidogenic enzyme HSD3B2. In conclusion, our studies in a mouse model with a human-like lipoprotein profile provide the first in vivo evidence for a novel inhibitory role of the LDLR in the control of adrenal glucocorticoid production.
\end{abstract}
Key Words
- cholesterol
- lipoprotein
- LDL
- receptor
- adrenal
- steroidogenesis
- corticosterone
- transplantation
- mice

Journal of Endocrinology (2015) 226, 145-154

\section{Introduction}

Cholesterol is the sole precursor for the synthesis of steroid hormones including adrenal-derived glucocorticoids, i.e., cortisol in man and corticosterone in rodents. The cholesterol used for steroidogenesis can theoretically be acquired from endogenous de novo synthesis of cholesterol, intracellular catabolism of stored cholesteryl esters, and the uptake of exogenous cholesterol carried by plasma lipoproteins (Kraemer 2007). It is generally assumed that the newly synthesized cholesterol pool is sufficient for basal glucocorticoid production by cells of the adrenal cortex. Upon stress-induced activation of the hypothalamic-pituitary-adrenal axis, the adrenals secrete high levels of glucocorticoids that mediate the physiological response needed to cope with stress through activation of

Published by Bioscientifica Ltd 
the glucocorticoid receptor (GR) in target tissues (Kadmiel \& Cidlowski 2013). We have previously shown that lipoprotein-associated cholesterol contributes at least $50 \%$ to the total cellular pool of cholesterol utilized for the production of glucocorticoids under high steroidogenic pressure conditions (Hoekstra et al. 2010).

Adrenals express high levels of scavenger receptor BI (SR-BI; Acton et al. 1996, Liu et al. 1997), the sole mediator of the selective cholesteryl ester uptake from HDLs (Out et al. 2004). In addition, expression of the LDL receptor (LDLR) has also been detected on glucocorticoid-producing cells of the adrenal zona fasciculata (Fong et al. 1989). In line with this, in vitro studies have suggested that both HDL and LDL fractions are capable of supplying cholesterol to the steroidogenic pool of cultured adrenocortical cells (Gwynne \& Hess 1980, Higashijima et al. 1987, Rainey et al. 1992, Yaguchi et al. 1998).

A genetic defect in proteins involved in the formation and cellular uptake of HDL-cholesteryl esters is associated with a diminished adrenal glucocorticoid output in experimental mouse models (Cai et al. 2008, Hoekstra et al. 2008, 2009) as well as in humans (Vergeer et al. 2011, Bochem et al. 2013). HDL-cholesterol thus appears to be of crucial importance for an optimal production of glucocorticoids by the adrenals in vivo.

A genetic deficiency of the LDLR does not alter the basal or stimulated corticosterone production by murine adrenocortical cells in vitro (Kraemer et al. 2007). Interestingly, homozygous human carriers of a mutation in the LDLR gene, familial hypercholesterolemia (FH) patients, do display a decrease in the maximal adrenal glucocorticoid output (Illingworth et al. 1983). However, no significant change in adrenal steroidogenesis rates has been detected in heterozygote human LDLR mutants (Illingworth et al. 1984). Importantly, lowering LDLcholesterol levels in FH patients by lovastatin (mevinolin) treatment does not further affect the adrenal glucocorticoid function (Laue et al. 1987). Furthermore, stimulation of the alternative LDL/LDLR route of cholesterol delivery to the adrenals by introducing human cholesteryl ester transfer protein in mice genetically lacking the HDL receptor SR-BI is also not able to reverse the associated glucocorticoid insufficiency (Hoekstra et al. 2009). Based on these combined findings, it can be suggested that the LDL/LDLR route does not play a major role in the supply of the steroidogenic cholesterol substrate in vivo. However, it should be noted that the lipoprotein profile in FH patients (hypercholesterolemic) and SR-BI-deficient mice (hyperalphalipoproteinemia) is markedly different from that of their respective normolipidemic controls, which makes proper interpretation of these data regarding the specific contribution of the adrenal LDL/LDLR route to steroidogenesis difficult. In the current study, therefore, we - by applying our adrenal transplantation technique - specifically modulated the adrenal LDLR genotype to determine the relative contribution of adrenocortical LDLR-mediated cholesterol uptake to the in vivo glucocorticoid function.

\section{Materials and methods}

\section{Adrenal transplantation}

Total body LDLR knockout mice crossed back to the C57BL/6 background (greater than eight generations) and C57BL/6 WT mice were bred in house. Adrenalectomy and subsequent adrenal transplantations were carried out as previously described (van der Sluis et al. 2012). At postnatal day 10, adrenal glands were isolated from female donor LDLR knockout or C57BL/6 pups to serve as transplant donors (Karpac et al. 2005). Eight- to 10-week-old female recipient LDLR knockout mice were bilaterally adrenalectomized under isoflurane inhalation anesthesia through a dorsal midline skin incision and lateral retroperitoneal incisions. Subsequently, one donor adrenal per recipient was placed under the kidney capsule through a slit in the renal capsule made by tweezers. Skin wounds were closed using michel suture clips. All mice were group-housed with three to five mice per cage and continuously supplied with normal chow diet, a $0.9 \% \mathrm{NaCl}$ solution, and normal water ad libitum. During the first week after surgery, a part of the bottom of each cage surface was heated by a heating mattress for optimal recovery from the operations. Based on our previous studies, adrenal transplants were given 8 weeks to become fully mature before subsequent transplant function and morphology analyses were executed. Fifteen of the $33 \mathrm{C} 57 \mathrm{BL} / 6$ and 12 of the 16 LDLR knockout recipient mice effectively completed the adrenal recovery period. At the end of the study, mice were subjected to overnight fasting to induce physiological stress, anesthetized, and killed. Subsequently, the arterial tree was perfused in situ with PBS (with the pressure of $100 \mathrm{mmHg}$ ) for $10 \mathrm{~min}$ via a cannula in the left ventricular apex, and organs were harvested, weighed, and either snap-frozen (RNA measurements) or stored in $3.7 \%$ formalin (tissue sectioning). No signs of endogenous adrenal regeneration were macroscopically visible in any of the transplanted mice. Animal experiments were performed in a temperature and light cycle $(12 \mathrm{~h}$ light: $12 \mathrm{~h}$ darkness) controlled room at the Gorlaeus Laboratories of the Leiden Academic Centre for Drug Research in

Published by Bioscientifica Ltd. 
accordance with the National Laws. All experimental protocols were approved by the Ethics Committee for Animal Experiments of Leiden University.

\section{Adrenal transplant glucocorticoid function tests}

Ad libitum-fed adrenal transplanted mice were bled at $0900 \mathrm{~h}$ via tail cut to measure basal plasma corticosterone levels on the lowest point of the circadian rhythm. Subsequently, the mice were administered a single dose of $200 \mu \mathrm{g}$ tetracosactide (adrenocorticotropic hormone (ACTH) fragment 1-24 human, rat; Sigma) intraperitoneally, followed by hourly blood draws up till $3 \mathrm{~h}$ post tetracosactide injection. To obtain a second measure of the maximal glucocorticoid output, adrenal recipient mice were bled by tail cut after food restriction from 1700 to $2100 \mathrm{~h}$ the next morning. During blood draws mice were handled identically and restrained for a maximum of $30 \mathrm{~s}$ to exclude an impact on blood corticosterone levels.

\section{Adrenal transplant histology}

Formalin-fixed cryosections $(8 \mu \mathrm{M})$ of adrenal transplants were prepared on a Leica CM3050-S cryostat (Leica Microsystems, Eindhoven, The Netherlands). Cryosections were routinely stained with hematoxylin and eosin or Oil Red O to visualize neutral lipids.

\section{Plasma measurements}

Corticosterone levels were determined using the Corticosterone Kit from MP Biomedicals (Irvine, CA, USA).
Concentrations of cholesterol were determined in blood of ad libitum-fed and overnight fasted mice using enzymatic colorimetric assays (Roche Diagnostics). The distribution of cholesterol over the different lipoprotein fractions in overnight fasting plasma was determined using a Superose 6 column $(3.2 \times 30 \mathrm{~mm}$, Smart System; Pharmacia), taking the efficiency of recovery from the column into account.

\section{Analysis of gene expression by real-time quantitative PCR}

Quantitative gene expression analysis on isolated adrenal transplants that were intact and free of surrounding fat and livers was performed as described (Hoekstra et al. 2003). In short, total RNA was isolated using a standard phenol/chloroform extraction method and reverse transcribed with RevertAid Reverse Transcriptase (Life Technologies). Gene expression analysis was performed using real-time SYBR Green Technology (Eurogentec, Maastricht, The Netherlands). Primers were validated for identical efficiencies. Primer sequences can be found in Table 1. Beta-actin (ACTB), glyceraldehyde-3-phosphate dehydrogenase, hypoxanthine phosphoribosyltransferase (HPRT), ATP synthase, $\mathrm{H}^{+}$transporting, mitochondrial Fo complex, subunit F6 (ATP5J), ribosomal protein L27 (RPL27), and acidic ribosomal phosphoprotein P0 (36B4) were used as the standard housekeeping genes. Relative expression levels were calculated using by subtracting the cycle threshold $(\mathrm{Ct})$ number from the gene of interest from the average housekeeping $C t$ and raising 2 to the power of this difference. No significant difference in the housekeeping $C t$ values was observed between the two adrenal transplant recipient groups.

Table 1 Primers used for real-time PCR analysis

\begin{tabular}{|c|c|c|}
\hline Gene & GenBank accession no. & Forward primer \\
\hline $36 B 4$ & NM007475 & 5'-CTGAGTACACCTTCCCACTTACTGA-3' \\
\hline$A C T B$ & NM007393 & 5'-AACCGTGAAAAGATGACCCAGAT-3' \\
\hline ACAT1 & NM144784 & 5'-AGCTGTTTCTCTGGGCCATCCAAT-3' \\
\hline ATP5J & NM016755 & $5^{\prime}-\mathrm{CAGGGCCGGAAGTAGAACGG-3^{ \prime }}$ \\
\hline CD14 & NM009841 & 5'-GGCGCTCCGAGTTGTGACT-3' \\
\hline CD68 & NM001291058 & 5'-TGCCTGACAAGGGACACTTCGGG-3' \\
\hline CD8 & NM009858 & 5'-GTGACTTCTACTTCTGCGCGAC-3' \\
\hline CYP11A1 & NM019779 & 5'-AGAACATCCAGGCCAACATTACCGAG-3' \\
\hline CYP11B1 & NM001033229 & 5'-GCCTGACCCGATGGACAA-3' \\
\hline CYP21A1 & NM009995 & 5'-GGGAACTGCCCAGCAAGTT-3' \\
\hline HPRT & NM013556 & $5^{\prime}$-TACAGCCCCAAAATGGTTAAGG-3' \\
\hline HSD3B2 & NM153193 & 5'-AGCCTTCCTGTGCCCCTACT-3' \\
\hline$H S L$ & NM010719 & $5^{\prime}$-CTGACAATAAAGGACTTGAGCAACTC- $3^{\prime}$ \\
\hline PEPCK & NM011044 & 5'-TTGAACTGACAGACTCGCCCT-3' \\
\hline RPL27 & NM011289 & $5^{\prime}$-CGCCAAGCGATCCAAGATCAAGTCC-3' \\
\hline STAR & NM011485 & 5'-GGGCATACTCAACAACCAGGAAGG-3' \\
\hline$S R-B I$ & NM016741 & 5'-GGCTGCTGTTTGCTGCG-3' \\
\hline TDO2 & NM019911 & $5^{\prime}$-ATGGCCATGTCAGGGATGA-3' \\
\hline
\end{tabular}

\begin{tabular}{l} 
Reverse primer \\
\hline 5'-CGACTCTTCCTTTGCTTCAGCTTT-3' \\
5'-CACAGCCTGGATGGCTACGTA-3'' \\
5'-GAACTCTCCTGGCTTCAGGGCAT-3' \\
5'-AAGGACAGAGGAGAGCCTGAAGA-3' \\
5'-GTACCTGCTTCAGCCCAGTGA-3' \\
5'-GCGGGTGATGCAGAAGGCGATG-3' \\
5'-AGGGTAGTCTTCTTGGTTGGGG-3' \\
5'-AGGACTTCAGCCCGCAGCATC-3' \\
5'-CAGAGATGAAATCCCAGGACTCTAA-3' \\
5'-AGGATGGTGTTCTGGGATTCTTC-3' \\
5'-AGTCAAGGGCATATCCAACAAC-3' \\
5'-CAGGAGGAAGCTCACAGTTTCC-3' \\
5'-AGGCCGCAGAAAAAAGTTGAC-3' \\
5'-GATATGCCCATCCGAGTCATG-3' \\
5'-AGCTGGGTCCCTGAACACATCCTTG-3' \\
5'-CTACCACCACCTCCAAGCGAAAC-3' \\
5'-GCTGCTTGATGAGGGAGGG-3' \\
5'-AAGATGACCACCACACGATGC-3'
\end{tabular}




\section{Statistical analysis}

Statistical analysis was performed using GraphPad Instat Software (San Diego, CA, USA, http://www.graphpad. com). Normality testing of the experimental groups was performed using the method of Kolmogorov and Smirnov. Significance was calculated using a two-tailed Student's t-test or two-way ANOVA with Bonferroni's post-test where appropriate. $P$ value $<0.05$ was considered significant.

\section{Results}

Our previous studies regarding the role of the HDL receptor SR-BI in the adrenal glucocorticoid function have indicated the value of our whole adrenal transplantation technique for studies regarding the contribution of adrenocortical cell-derived transcripts to glucocorticoid homeostasis (Hoekstra et al. 2013). To determine the impact of the LDLR located on adrenocortical cells for glucocorticoid production in vivo, in this study, we therefore transplanted either one C57BL/6 adrenal carrying a functional LDLR (LDLR positive (LDLR+)) or one adrenal genetically lacking the LDLR (LDLR negative $(\mathrm{LDLR}-)$ ) under the renal capsule of recipient mice. We anticipate that the importance of the LDL/LDLR pathway of cholesterol delivery in adrenals of normolipidemic WT mice - as opposed to humans - is limited as virtually no LDL-associated cholesterol can be detected in plasma of these mice due to rapid clearance of LDL particles by the LDLR located on hepatocytes within the liver. In the current experimental setup we therefore specifically chose to transplant adrenals of either genotype into adrenalectomized total body LDLR knockout recipient mice that exhibit a human-like plasma lipoprotein profile, i.e. with relatively high LDL-cholesterol levels, due to an impaired hepatic clearance of LDL particles (Ishibashi et al. 1993).

In accordance with accumulation of cholesterol in the blood compartment as a result of defective clearance by the liver, total cholesterol levels were significantly higher in plasma of both types of ad libitum-fed LDLR knockout recipient mice ( $\sim 200-250 \mathrm{mg} / \mathrm{dl}$; Fig. 1 ) as compared to those generally found in WT mice (60-100 mg/dl; Van Eck et al. 2003, Out et al. 2007). Subjecting mice to food deprivation stimulates a stress response that involves an ACTH-mediated activation of adrenal glucocorticoid production (Chida et al. 2007). Fasting the mice overnight had no significant effect on plasma total cholesterol levels (Fig. 1; two-way ANOVA: $P>0.05$ ). Fasting free cholesterol levels were unchanged by the adrenal genotype $(110 \pm 12 \mathrm{mg} / \mathrm{dl}$ for $\mathrm{LDLR}+$ adrenal recipients vs
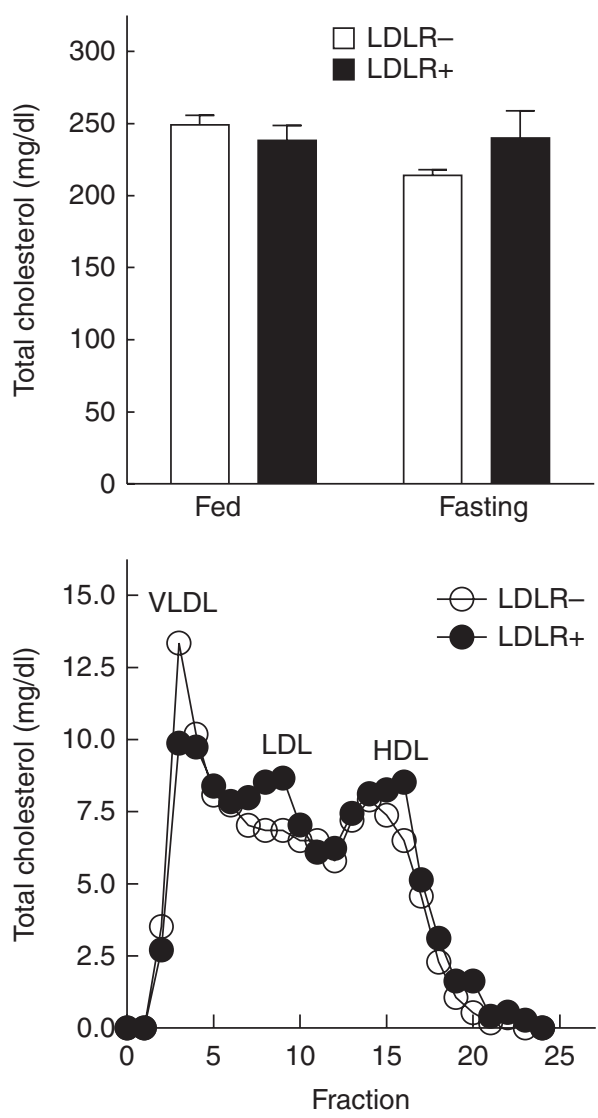

Figure 1

Effect of the adrenal LDL receptor (LDLR) genotype on plasma cholesterol levels. Mice were bled in the ad libitum-fed state (fed) or after overnight food deprivation (fasting). Data represent means \pm s.E.M. of 12 LDLR knockout mice that genetically lack the LDLR in their adrenal transplants (LDLR -) and 15 LDLR knockout mice that were transplanted with WT adrenals containing functional LDLRs (LDLR + ). Pooled plasma of the different adrenal recipients was used to generate the lipoprotein distribution profiles. VLDL, very-LDL.

$90 \pm 3 \mathrm{mg} / \mathrm{dl}$ for LDLR - adrenal recipients; $P>0.05)$. In addition, the adrenal transplant genotype did not impact on total cholesterol levels either in the fasting or ad libitumfed state (Fig. 1; two-way ANOVA: P>0.05). Two-way ANOVA also did not reveal a significant interaction $(P>0.05)$ between the adrenal transplant genotype and feeding status. As evident from Fig. 1, the distribution of cholesterol over the different lipoprotein fractions was also virtually identical in the two groups of recipients under fasting stress conditions, with the majority of cholesterol being transported in the very-LDL (VLDL) and LDL fractions. These primary findings suggest i) a negligible contribution of LDLR-mediated uptake of cholesterol by the adrenals to the regulation of plasma cholesterol levels and ii) that a possible difference in adrenocortical function

Published by Bioscientifica Ltd 

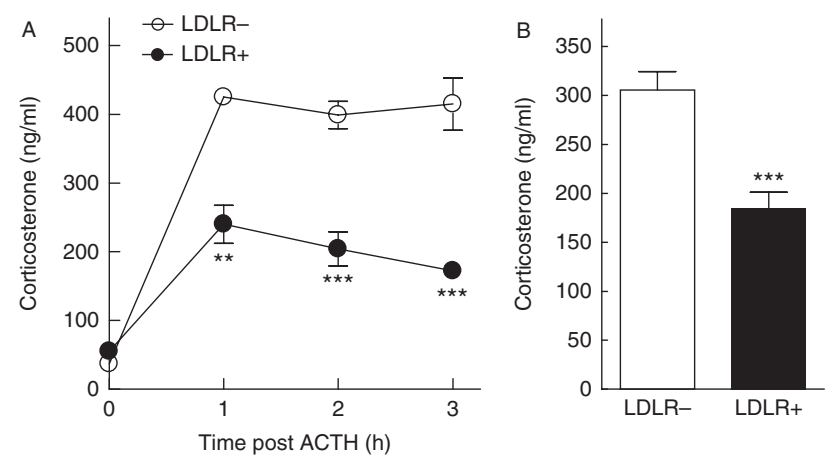

Figure 2

Effect of the adrenal LDL receptor genotype on plasma glucocorticoid levels. (A) Time profile of plasma corticosterone levels upon i.p. injection of ACTH. Two-way ANOVA: $P<0.001$ for time; $P<0.001$ for adrenal genotype; and $P<0.001$ for interaction. (B) Corticosterone levels in overnight fasted mice. Data represent means \pm s.E.M. of four to nine mice (ACTH response) and 13-15 mice (fasting) per group. ${ }^{*} P<0.01, * * * P<0.001$ vs LDLR - .

between the experimental groups is not secondary to a shift in lipoprotein-associated cholesterol (substrate) availability.

In line with the general notion that the lipoprotein/ lipoprotein receptor system is not of quantitative importance for the control of basal adrenal steroidogenesis, plasma levels of corticosterone were not different between the two adrenocortical LDLR genotypes in the ad libitum fed non-stressed state (Fig. 2). LDLR - adrenal transplant recipient mice displayed a rapid increase in circulating levels of corticosterone upon i.p. injection of a synthetic analog of the pituitary-derived steroidogenic activator ACTH (tetracosactide), indicative of an efficient recovery of the adrenal steroid function after adrenal transplantation. Importantly, as can be appreciated from Fig. 2, restoration of adrenal LDLR function in total body LDLR knockout mice led to a marked reduction in the ACTHmediated stimulation of adrenal steroidogenesis (two-way ANOVA: $P<0.001$ for genotype), with plasma corticosterone levels that were respectively $44-59 \%$ lower $(P<0.01)$ as compared to adrenal LDLR - controls. In addition, LDLR + adrenal transplanted mice exhibited a significant decrease $(-39 \% ; P<0.001)$ in their plasma corticosterone level under fasting stress conditions. Normalization of the adrenal LDLR function was thus paralleled by a diminished glucocorticoid output both in response to ACTH stimulation and under fasting stress conditions.

Glucocorticoids activate their cognate GR to execute immunosuppressive effects in lymphoid organs, while stimulating the synthesis and utilization of glucose in metabolic tissues (Kadmiel \& Cidlowski 2013). In our study, thymus weights did not differ between the two adrenocortical genotypes under fasting stress conditions (Fig. 3), suggesting the presence of an equally effective glucocorticoid response to induce acute apoptosis in thymocytes. In contrast, we did note an increase in relative spleen weights $(+34 \% ; P<0.05$; Fig. 3$)$ pointing to a long-term impairment of overall glucocorticoid action in splenocytes in response to normalization of the adrenocortical LDLR function. In further support of general glucocorticoid insufficiency upon replenishment of adrenal LDLR function, hepatic transcript levels of the glucocorticoid-responsive genes phosphoenolpyruvate carboxykinase (PEPCK) and tryptophan 2,3-dioxygenase (TDO2) were respectively 53\% $(P<0.01)$ and $33 \%$ $(P<0.01)$ lower in LDLR + as compared to LDLR - adrenal transplanted mice under fasting stress conditions (Fig. 4).

We performed gene expression analysis on the six LDLR + adrenal transplants and five LDLR - adrenal transplants that could be isolated microscopically intact and virtually free of adjacent kidney tissue to uncover the (molecular) mechanism underlying the diminished glucocorticoid output under fasting stress conditions. To exclude that the reduction in steroidogenic capacity was secondary to a host vs graft response directed against the LDLR that was introduced on adrenocortical cells through transplantation, we measured relative mRNA expression level of inflammatory cell markers (Fig. 5A). Basal mRNA expression levels of the respective cytotoxic $\mathrm{T}$ cell, monocyte, and macrophage markers CD8 (Ct: $32.9 \pm$

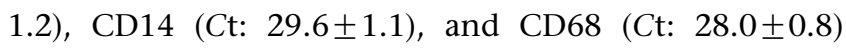
were low in control LDLR - adrenal transplants and not significantly higher in transplants derived from LDLR
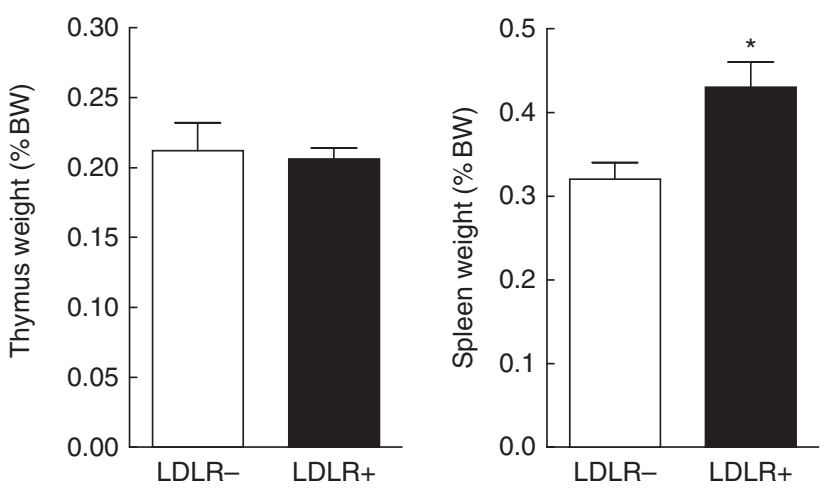

Figure 3

Effect of the adrenal LDL receptor genotype on thymus and spleen weights. Respective tissue weights are derived from mice subjected to overnight fasting and expressed as percentage of the total body weight (\%BW). Data represent means \pm s.E.M. of $12-14$ mice/group. ${ }^{*} P<0.05$ vs LDLR - .

Published by Bioscientifica Ltd 

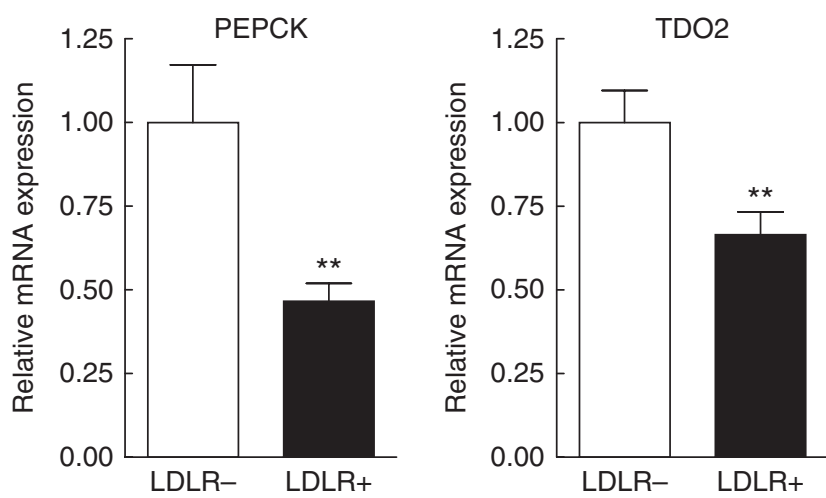

Figure 4

Effect of the adrenal LDL receptor genotype on hepatic relative expression levels of glucocorticoid receptor target genes. Gene expression was measured in livers isolated from mice that had been subjected to overnight fasting. Data represent means \pm s.E.M. of $8-12$ mice/group. ${ }^{* *} P<0.01$ vs LDLR-.

expressing adrenal recipients. Combined with the fact that hematoxylin and eosin-stained slides (Fig. 5B) of both adrenal transplant subtypes did not show the immune cell infiltrates that are typically seen in graft vs host specimens, it is suggested that an immune response directed against the LDLR likely does not explain the inhibitory effect on adrenal steroidogenesis. In accordance with a stress-induced stimulation of the steroidogenic machinery, expression of rate-limiting enzymes involved in the mobilization of cholesterol, such as SR-BI (Ct: 21.4 \pm 1.1$)$ and STAR (Ct: $23.2 \pm 1.1$ ), and subsequent conversion into corticosterone, i.e., CYP11A1 (Ct: $20.9 \pm 0.9$ ), HSD3B2 (Ct: $26.3 \pm 0.9$ ), CYP21A1 (Ct: $21.6 \pm 0.7)$, and CYP11B1 (Ct: $25.4 \pm 1.3)$, could be readily detected in LDLR adrenal transplants under fasting conditions. Stimulation of the expression of the HDL receptor SR-BI is an obligatory event in the ACTH-mediated adrenal stress response to maintain functional adrenal cholesterol stores (Rigotti et al. 1996, Hoekstra et al. 2008, 2009, 2013). Interestingly, as can be seen in Fig. 6, the LDLR genotype did not impact on the adrenal transplant relative mRNA expression levels of SR-BI. It can therefore be suggested that the rate of HDL-associated cholesterol ester uptake into adrenals and the efficacy of the adrenocortical cell ACTH signaling was not different in the two groups of adrenal recipients. The expression of genes associated with the respective formation and catabolism of cholesteryl esters, acetyl-CoA acetyltransferase 1 (ACAT1) and hormone-sensitive lipase (HSL), was also not affected by the adrenal LDLR genotype (Fig. 6). In addition, we detected a similar relative mRNA expression level of the STAR protein that delivers cholesterol to the inner mitochondrial membrane for subsequent use in the steroidogenic pathway (Fig. 6). It thus appears that the change in maximal steroidogenesis rate in response to a change in adrenal LDLR genotype could not be attributed to an altered (intracellular) mobilization of cholesterol. In accordance, Oil Red O lipid neutral lipid staining revealed that the two adrenal transplant types both contained high amounts of cholesterol under fasting stress conditions (Fig. 7). No significant change in the relative mRNA expression levels of mitochondrial cholesterol side-chain cleavage enzyme (CYP11A1), steroid 21-hydroxylase (CYP21A1), or steroid11-beta-hydroxylase (CYP11B1) was noted. In contrast, LDLR + adrenal transplants displayed a marked $62 \%$ reduction $(P<0.05$; Fig. 6$)$ in the transcript level of $3 \beta$-hydroxysteroid dehydrogenase/ $\Delta 5-\Delta 4$-isomerase type 2 (HSD3B2) that executes the second step in the steroidogenesis pathway, i.e., the catabolism of pregnenolone into progesterone. These combined findings suggest that the reduced fasting glucocorticoid response associated with restoration of adrenocortical LDLR function may be secondary to a diminished adrenal HSD3B2 activity.

A

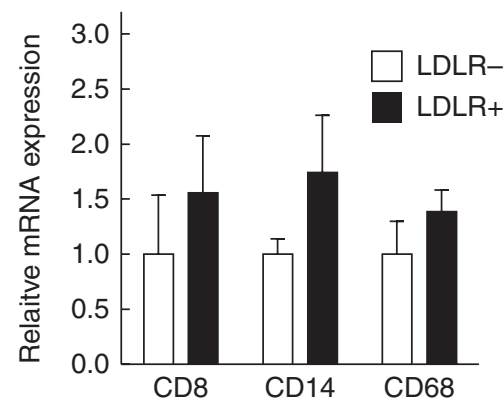

B

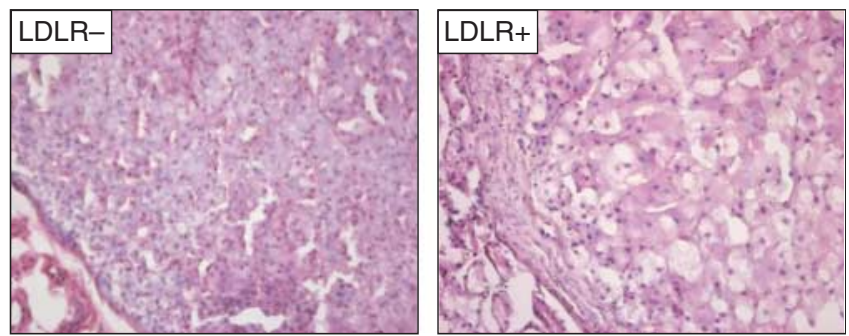

Figure 5

Effect of the adrenal LDL receptor genotype on adrenal transplant inflammation status. (A) Gene expression of inflammatory cell markers was measured in adrenal transplants isolated from mice that had been subjected to overnight fasting. Data represent means \pm s.E.M. of five to six mice per group. (B) Representative adrenal transplant sections were stained with hematoxylin and eosin. Clear immune cell infiltrates are absent in both adrenal transplant groups. Original magnification, $200 \times$. A full colour version of this figure is available at http://dx.doi.org/10.1530/ JOE-15-0023.

Published by Bioscientifica Ltd 

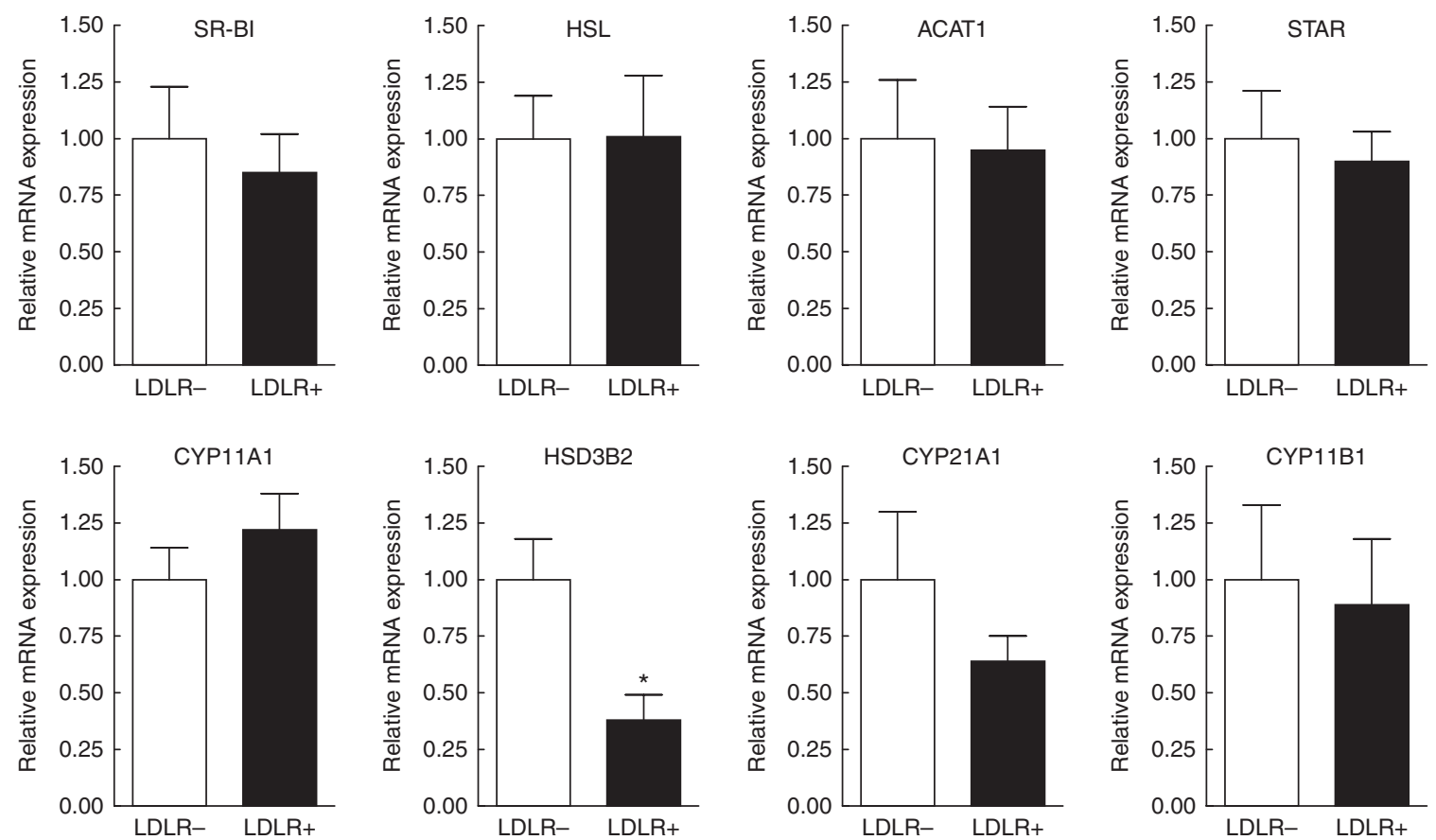

\section{Figure 6}

Effect of the adrenal LDL receptor genotype on adrenal transplant relative expression levels of genes associated with cholesterol mobilization and steroidogenesis. Gene expression was measured in adrenal transplants

\section{Discussion}

Although several lines of evidence have suggested that the LDLR can supply cholesterol to the steroidogenic pathway for subsequent conversion to glucocorticoids, our current findings clearly indicate that - in mice with a human-like lipoprotein profile - the maximal glucocorticoid output is negatively influenced by the presence of the LDLR in adrenocortical cells.

Apolipoprotein E (APOE) is essential for the interaction of lipoprotein particles with the LDLR (Bradley \& Gianturco 1986). Owing to a diminished LDLR-mediated clearance, APOE knockout mice display marked hypercholesterolemia, i.e., accumulation of APOB-containing lipoproteins in plasma (Zhang et al. 1992). Importantly, APOE knockout mice also show an increase in both basal and stress-induced glucocorticoid levels (Raber et al. 2000, Grootendorst et al. 2002, 2004, Thorngate et al. 2002), which provides further in vivo support for a causal inverse relationship between LDLR-mediated cholesterol uptake and glucocorticoid output.

Although most studies on the effect of lipoproteins on adrenal function have focused on steroidogenesis, it should be acknowledged that cholesterol is essential for

adrenal growth in vivo (Dominick et al. 1993). Based on the fact that LDLRs on adrenocortical cells are able to effectively clear cholesterol-rich LDL particles upon ACTH administration in vivo (Kovanen et al. 1980), we anticipate that the cholesterol derived from LDL is rather used for non-steroidogenic adrenal functions, including adrenocortical cell proliferation. This notion is further substantiated by the finding of Kraemer et al. (2007) that
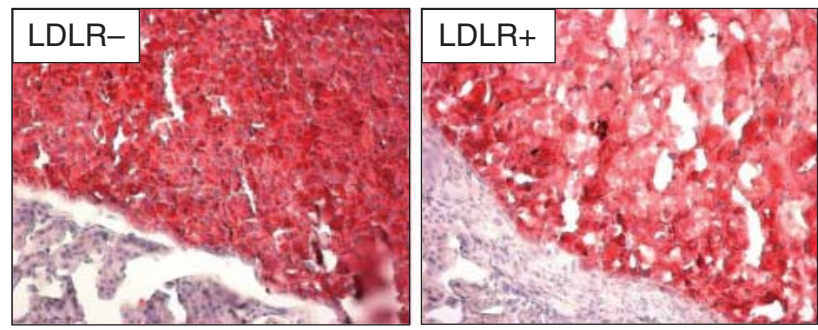

Figure 7

Effect of the adrenal LDL receptor genotype on adrenal transplant cholesterol stores. Adrenal transplants were isolated from mice that had been subjected to overnight fasting. Representative adrenal transplant sections were stained for neutral lipids using Oil Red O. Original magnification, $200 \times$. A full colour version of this figure is available at http://dx.doi.org/10.1530/JOE-15-0023. 
the LDLR genotype of adrenocortical cells has no impact on the ACTH-stimulated steroidogenic response in vitro.

The decrease in maximal glucocorticoid secretion observed upon replenishment of adrenocortical LDLR function in total body LDLR knockout mice coincided with a decrease in the relative expression level of the steroidogenic enzyme HSD3B2. Importantly, genetic HSD3B2 deficiency represents an established, but rare, cause of congenital adrenal hyperplasia (CAH) in humans. Homozygous carriers of a functional mutation in HSD3B2 display significantly lower cortisol levels and salt-wasting (Alos et al. 2000, Jeandron \& Sahakitrungruang 2012). We therefore anticipate that the glucocorticoid insufficiency phenotype associated with restoration of the LDLR function can be directly attributed to the decrease in adrenocortical HSD3B2 expression.

A literature search did not reveal a direct link between adrenocortical LDLR function, LDL-cholesterol uptake, and the regulation of $\mathrm{HSD} 3 \mathrm{~B} 2$ mRNA expression. Although other transcription factors such as GATA family members and the nuclear receptor liver receptor homolog 1 (NR5A2) are also able to modulate the promoter activity of HSD3B2, it has been established that the growth-factorinducible immediate early gene nur/77 (NUR77) and steroidogenic factor 1 (SF1 (NR5A1)) are essential for maintaining basal HSD3B2 transcription in human adrenocortical cells (Leers-Sucheta et al. 1997, Martin \& Tremblay 2005, Udhane et al. 2013). The relative mRNA expression level of NUR77 and SF1 was not significantly different between our two adrenal transplants (data not shown). The LDLR genotype thus does not directly impact on the expression level of critical upstream regulators of HSD3B2. The decrease in adrenal HSD3B2 expression due to normalization of LDLR function might, however, be secondary to a change in the activity of intracellular signaling pathways. The anti-cancer drug sunitinib lowers the secretion of cortisol by cultured human NCI-H295 adrenocortical cells, which can be attributed specifically to a decrease in the mRNA and protein expression of HSD3B2 (Kroiss et al. 2011). The small molecule sunitinib is a potent multi-targeted receptor tyrosine kinase inhibitor (O'Farrell et al. 2003, Sun et al. 2003), suggesting that changes in intracellular signaling pathways are able to modulate the HSD3B2 transcription rate. Studies in respectively cultured mesangial cells (Jenkins et al. 2000) and osteoblasts (Klein et al. 2006) have indicated that exposure to native LDL - the normal substrate of the LDLR - increases the activity (phosphorylation state) of MAPK and tuberin and lowers Src protein levels and Akt activity. These combined findings suggest that, by changing the activity of major intracellular signaling pathways, enhanced adrenal uptake of native LDL from the plasma compartment might ultimately decrease the HSD3B2 expression in this tissue.

In conclusion, we have shown that restoration of adrenal LDLR function in LDLR knockout mice is associated with a diminished glucocorticoid output and signaling. Our studies in a mouse model with a humanlike lipoprotein profile i) provide the first in vivo evidence for a novel inhibitory role of the LDLR in the control of adrenal steroidogenesis and ii) highlight that the relative impact of specific lipoprotein cholesterol sources and their receptors on adrenocortical cell-mediated steroidogenesis may depend much on the overall substrate availability and complexity of the study system, i.e., in vitro cell cultures vs total body and tissue-specific knockout mouse models or humans.

\section{Declaration of interest}

All authors have nothing to disclose. Our grant suppliers, the Dutch Heart Foundation and Netherlands Organization for Scientific Research, are noncommercial funding agencies and were not involved in the acquisition and interpretation of the data or the decision to publish the work.

\section{Funding}

This work was supported by grants $2008 \mathrm{~T} 070$ and 2012T080 from the Dutch Heart Foundation awarded to $\mathrm{M} \mathrm{H}$ and $\mathrm{VICl}$ grant 91813603 from the Netherlands Organization for Scientific Research awarded to M V E. M V E is an Established Investigator of the Dutch Heart Foundation (grant number 2007T056).

\section{Author contribution statement}

$\mathrm{R} \mathrm{J} \vee \mathrm{d} \mathrm{S}$ and $\mathrm{M} \mathrm{H}$ executed the experiments and performed data analysis. $M V E$ is supervisor of $R J \vee d S$ and head of the lipid group within the Division of Biopharmaceutics and thereby facilitated all aspects of the research. $\mathrm{M} \mathrm{H}$ wrote the manuscript.

\section{References}

Acton S, Rigotti A, Landschulz KT, Xu S, Hobbs HH \& Krieger M 1996 Identification of scavenger receptor SR-BI as a high density lipoprotein receptor. Science 271 518-520. (doi:10.1126/science.271.5248.518)

Alos N, Moisan AM, Ward L, Desrochers M, Legault L, Leboeuf G, Van Vliet G \& Simard J 2000 A novel A10E homozygous mutation in the HSD3B2 gene causing severe salt-wasting $3 \beta$-hydroxysteroid dehydrogenase deficiency in 46,XX and 46,XY French-Canadians: evaluation of gonadal function after puberty. Journal of Clinical Endocrinology and Metabolism 85 1968-1974. (doi:10.1210/jcem.85.5.6581)

Bochem AE, Holleboom AG, Romijn JA, Hoekstra M, Dallinga-Thie GM, Motazacker MM, Hovingh GK, Kuivenhoven JA \& Stroes ES 2013 High density lipoprotein as a source of cholesterol for adrenal steroidogenesis: a study in individuals with low plasma HDL-C. Journal of Lipid Research 54 1698-1704. (doi:10.1194/jlr.P033449)

Published by Bioscientifica Ltd 
Bradley WA \& Gianturco SH 1986 ApoE is necessary and sufficient for the binding of large triglyceride-rich lipoproteins to the LDL receptor; apoB is unnecessary. Journal of Lipid Research 27 40-48.

Cai L, Ji A, de Beer FC, Tannock LR \& van der Westhuyzen DR 2008 SR-BI protects against endotoxemia in mice through its roles in glucocorticoid production and hepatic clearance. Journal of Clinical Investigation 118 364-375. (doi:10.1172/JCI31539)

Chida D, Nakagawa S, Nagai S, Sagara H, Katsumata H, Imaki T, Suzuki H, Mitani F, Ogishima T, Shimizu C et al. 2007 Melanocortin 2 receptor is required for adrenal gland development, steroidogenesis, and neonatal gluconeogenesis. PNAS 104 18205-18210. (doi:10.1073/pnas. 0706953104)

Dominick MA, Bobrowski WA, MacDonald JR \& Gough AW 1993 Morphogenesis of a zone-specific adrenocortical cytotoxicity in guinea pigs administered PD 132301-2, an inhibitor of acyl-CoA:cholesterol acyltransferase. Toxicologic Pathology 21 54-62. (doi:10.1177/ 019262339302100107)

Fong LG, Bonney E, Kosek JC \& Cooper AD 1989 Immunohistochemical localization of low density lipoprotein receptors in adrenal gland, liver, and intestine. Journal of Clinical Investigation 84 847-856. (doi:10.1172/ JCI114245)

Grootendorst J, Kempes MM, Lucassen PJ, Dalm S, de Kloet ER \& Oitzl MS 2002 Differential effect of corticosterone on spatial learning abilities in apolipoprotein E knockout and C57BL/6J mice. Brain Research 953 281-285. (doi:10.1016/S0006-8993(02)03399-1)

Grootendorst J, Enthoven L, Dalm S, de Kloet ER \& Oitzl MS 2004 Increased corticosterone secretion and early-onset of cognitive decline in female apolipoprotein E-knockout mice. Behavioural Brain Research 148 167-177. (doi:10.1016/S0166-4328(03)00188-8)

Gwynne JT \& Hess B 1980 The role of high density lipoproteins in rat adrenal cholesterol metabolism and steroidogenesis. Journal of Biological Chemistry 255 10875-10883.

Higashijima M, Kato K, Nawata H \& Ibayashi H 1987 Studies on lipoprotein and adrenal steroidogenesis: II. Utilization of low density lipoproteinand high density lipoprotein-cholesterol for steroid production in functioning human adrenocortical adenoma cells in culture. Endocrinologia Japonica 34 647-657. (doi:10.1507/endocrj1954.34.647)

Hoekstra M, Kruijt JK, Van Eck M \& Van Berkel TJ 2003 Specific gene expression of ATP-binding cassette transporters and nuclear hormone receptors in rat liver parenchymal, endothelial, and Kupffer cells. Journal of Biological Chemistry 278 25448-25453. (doi:10.1074/jbc. M301189200)

Hoekstra M, Meurs I, Koenders M, Out R, Hildebrand RB, Kruijt JK, Van Eck M \& Van Berkel TJ 2008 Absence of HDL cholesteryl ester uptake in mice via SR-BI impairs an adequate adrenal glucocorticoid-mediated stress response to fasting. Journal of Lipid Research 49 738-745. (doi:10.1194/jlr.M700475-JLR200)

Hoekstra M, Ye D, Hildebrand RB, Zhao Y, Lammers B, Stitzinger M, Kuiper J, Van Berkel TJ \& Van Eck M 2009 Scavenger receptor class B type I-mediated uptake of serum cholesterol is essential for optimal adrenal glucocorticoid production. Journal of Lipid Research $\mathbf{5 0}$ 1039-1046. (doi:10.1194/jlr.M800410-JLR200)

Hoekstra M, Korporaal SJ, Li Z, Zhao Y, Van Eck M \& Van Berkel TJ 2010 Plasma lipoproteins are required for both basal and stress-induced adrenal glucocorticoid synthesis and protection against endotoxemia in mice. American Journal of Physiology. Endocrinology and Metabolism 299 E1038-E1043. (doi:10.1152/ajpendo.00431.2010)

Hoekstra M, van der Sluis RJ, Van Eck M \& Van Berkel TJ 2013 Adrenal-specific scavenger receptor BI deficiency induces glucocorticoid insufficiency and lowers plasma very-low-density and low-density lipoprotein levels in mice. Arteriosclerosis, Thrombosis, and Vascular Biology 33 e39-e46. (doi:10.1161/ATVBAHA. 112.300784)

Illingworth DR, Lees AM \& Lees RS 1983 Adrenal cortical function in homozygous familial hypercholesterolemia. Metabolism 32 1045-1052. (doi:10.1016/0026-0495(83)90075-6)
Illingworth DR, Alam NA \& Lindsey S 1984 Adrenocortical response to adrenocorticotropin in heterozygous familial hypercholesterolemia. Journal of Clinical Endocrinology and Metabolism 58 206-211. (doi:10.1210/jcem-58-1-206)

Ishibashi S, Brown MS, Goldstein JL, Gerard RD, Hammer RE \& Herz J 1993 Hypercholesterolemia in low density lipoprotein receptor knockout mice and its reversal by adenovirus-mediated gene delivery. Journal of Clinical Investigation 92 883-893. (doi:10.1172/JCI116663)

Jeandron DD \& Sahakitrungruang T 2012 A novel homozygous Q334X mutation in the HSD3B2 gene causing classic 3 $\beta$-hydroxysteroid dehydrogenase deficiency: an unexpected diagnosis after a positive newborn screen for 21-hydroxylase deficiency. Hormone Research in Paediatrics 77 334-348. (doi:10.1159/000336004)

Jenkins AJ, Velarde V, Klein RL, Joyce KC, Phillips KD, Mayfield RK, Lyons TJ \& Jaffa AA 2000 Native and modified LDL activate extracellular signal-regulated kinases in mesangial cells. Diabetes 49 2160-2169. (doi:10.2337/diabetes.49.12.2160)

Kadmiel M \& Cidlowski JA 2013 Glucocorticoid receptor signaling in health and disease. Trends in Pharmacological Sciences 34 518-530. (doi:10.1016/j.tips.2013.07.003)

Karpac J, Ostwald D, Bui S, Hunnewell P, Shankar M \& Hochgeschwender U 2005 Development, maintenance, and function of the adrenal gland in early postnatal proopiomelanocortin-null mutant mice. Endocrinology 146 2555-2562. (doi:10.1210/en.2004-1290)

Klein BY, Kerem Z \& Rojansky N 2006 LDL induces Saos2 osteoblasts death via Akt pathways responsive to a neutral sphingomyelinase inhibitor. Journal of Cellular Biochemistry 98 661-671. (doi:10.1002/jcb.20807)

Kovanen PT, Goldstein JL, Chappell DA \& Brown MS 1980 Regulation of low density lipoprotein receptors by adrenocorticotropin in the adrenal gland of mice and rats in vivo. Journal of Biological Chemistry $\mathbf{2 5 5}$ 5591-5598.

Kraemer FB 2007 Adrenal cholesterol utilization. Molecular and Cellular Endocrinology 265-266 42-45. (doi:10.1016/j.mce.2006.12.001)

Kraemer FB, Shen WJ, Patel S, Osuga J, Ishibashi S \& Azhar S 2007 The LDL receptor is not necessary for acute adrenal steroidogenesis in mouse adrenocortical cells. American Journal of Physiology. Endocrinology and Metabolism 292 E408-E412. (doi:10.1152/ajpendo.00428.2006)

Kroiss M, Reuss M, Kühner D, Johanssen S, Beyer M, Zink M, Hartmann MF, Dhir V, Wudy SA, Arlt W et al. 2011 Sunitinib inhibits cell proliferation and alters steroidogenesis by down-regulation of HSD3B2 in adrenocortical carcinoma cells. Frontiers in Endocrinology 2 27. (doi:10.3389/ fendo.2011.00027)

Laue L, Hoeg JM, Barnes K, Loriaux DL \& Chrousos GP 1987 The effect of mevinolin on steroidogenesis in patients with defects in the low density lipoprotein receptor pathway. Journal of Clinical Endocrinology and Metabolism 64 531-535. (doi:10.1210/jcem-64-3-531)

Leers-Sucheta S, Morohashi K, Mason JI \& Melner MH 1997 Synergistic activation of the human type II $3 \beta$-hydroxysteroid dehydrogenase/ $\Delta 5-\Delta 4$ isomerase promoter by the transcription factor steroidogenic factor-1/adrenal 4-binding protein and phorbol ester. Journal of Biological Chemistry 272 7960-7967. (doi:10.1074/jbc.272.12.7960)

Liu J, Voutilainen R, Heikkilä P \& Kahri AI 1997 Ribonucleic acid expression of the CLA-1 gene, a human homolog to mouse high density lipoprotein receptor SR-BI, in human adrenal tumors and cultured adrenal cells. Journal of Clinical Endocrinology and Metabolism 82 2522-2527. (doi:10.1210/jcem.82.8.4123)

Martin LJ \& Tremblay JJ 2005 The human 3 $\beta$-hydroxysteroid dehydrogenase/ $\Delta 5-\Delta 4$ isomerase type 2 promoter is a novel target for the immediate early orphan nuclear receptor Nur77 in steroidogenic cells. Endocrinology 146 861-869. (doi:10.1210/en.2004-0859)

O'Farrell AM, Abrams TJ, Yuen HA, Ngai TJ, Louie SG, Yee KW, Wong LM, Hong W, Lee LB, Town A et al. 2003 SU11248 is a novel FLT3 tyrosine kinase inhibitor with potent activity in vitro and in vivo. Blood $\mathbf{1 0 1}$ 3597-3605. (doi:10.1182/blood-2002-07-2307)

Out R, Hoekstra M, Spijkers JA, Kruijt JK, van Eck M, Bos IS, Twisk J \& Van Berkel TJ 2004 Scavenger receptor class B type I is solely responsible

Published by Bioscientifica Ltd. 
for the selective uptake of cholesteryl esters from HDL by the liver and the adrenals in mice. Journal of Lipid Research 45 2088-2095. (doi:10.1194/jlr.M400191-JLR200)

Out R, Hoekstra M, Meurs I, de Vos P, Kuiper J, Van Eck M \& Van Berkel TJ 2007 Total body ABCG1 expression protects against early atherosclerotic lesion development in mice. Arteriosclerosis, Thrombosis, and Vascular Biology 27 594-599. (doi:10.1161/01.ATV.0000257136. 24308.0c)

Raber J, Akana SF, Bhatnagar S, Dallman MF, Wong D \& Mucke L 2000 Hypothalamic-pituitary-adrenal dysfunction in Apoe $(-/-)$ mice: possible role in behavioral and metabolic alterations. Journal of Neuroscience 20 2064-2071.

Rainey WE, Rodgers RJ \& Mason JI 1992 The role of bovine lipoproteins in the regulation of steroidogenesis and HMG-CoA reductase in bovine adrenocortical cells. Steroids 57 167-173. (doi:10.1016/0039128X(92)90003-R)

Rigotti A, Edelman ER, Seifert P, Iqbal SN, DeMattos RB, Temel RE, Krieger M \& Williams DL 1996 Regulation by adrenocorticotropic hormone of the in vivo expression of scavenger receptor class B type I (SR-BI), a high density lipoprotein receptor, in steroidogenic cells of the murine adrenal gland. Journal of Biological Chemistry 271 33545-33549. (doi:10.1074/ jbc.271.52.33545)

van der Sluis RJ, van Puijvelde GH, Van Berkel TJ \& Hoekstra M 2012 Adrenalectomy stimulates the formation of initial atherosclerotic lesions: reversal by adrenal transplantation. Atherosclerosis 221 76-83. (doi:10.1016/j.atherosclerosis.2011.12.022)

Sun L, Liang C, Shirazian S, Zhou Y, Miller T, Cui J, Fukuda JY, Chu JY, Nematalla A, Wang X et al. 2003 Discovery of 5-[5-fluoro-2-oxo-1,2dihydroindol-(3Z)-ylidenemethyl]-2,4-dimethyl-1H-pyrrole-3-carboxylic acid (2-diethylaminoethyl)amide, a novel tyrosine kinase inhibitor targeting vascular endothelial and platelet-derived growth factor receptor tyrosine kinase. Journal of Medicinal Chemistry 46 1116-1119. (doi:10.1021/jm0204183)

Thorngate FE, Strockbine PA, Erickson SK \& Williams DL 2002 Altered adrenal gland cholesterol metabolism in the apoE-deficient mouse. Journal of Lipid Research 43 1920-1926. (doi:10.1194/jlr.M200205JLR200)

Udhane S, Kempna P, Hofer G, Mullis PE \& Flück CE 2013 Differential regulation of human $3 \beta$-hydroxysteroid dehydrogenase type 2 for steroid hormone biosynthesis by starvation and cyclic AMP stimulation: studies in the human adrenal NCI-H295R cell model. PLoS ONE 8 e68691. (doi:10.1371/journal.pone.0068691)

Van Eck M, Twisk J, Hoekstra M, Van Rij BT, Van der Lans CA, Bos IS, Kruijt JK, Kuipers F \& Van Berkel TJ 2003 Differential effects of scavenger receptor BI deficiency on lipid metabolism in cells of the arterial wall and in the liver. Journal of Biological Chemistry $\mathbf{2 7 8}$ 23699-23705. (doi:10.1074/jbc.M211233200)

Vergeer M, Korporaal SJ, Franssen R, Meurs I, Out R, Hovingh GK, Hoekstra M, Sierts JA, Dallinga-Thie GM, Motazacker MM et al. 2011 Genetic variant of the scavenger receptor BI in humans. New England Journal of Medicine 364 136-1345. (doi:10.1056/NEJMoa0907687)

Yaguchi H, Tsutsumi K, Shimono K, Omura M, Sasano H \& Nishikawa T 1998 Involvement of high density lipoprotein as substrate cholesterol for steroidogenesis by bovine adrenal fasciculo-reticularis cells. Life Sciences 62 1387-1395. (doi:10.1016/S0024-3205(98)00077-0)

Zhang SH, Reddick RL, Piedrahita JA \& Maeda N 1992 Spontaneous hypercholesterolemia and arterial lesions in mice lacking apolipoprotein E. Science $\mathbf{2 5 8}$ 468-471. (doi:10.1126/science.1411543)

Received in final form 26 June 2015

Accepted 1 July 2015

Accepted Preprint published online 1 July 2015
(C) 2015 Society for Endocrinology Printed in Great Britain 